\title{
GENERATIONSKONFLIKT UND ZEITENWECHSEL IN DER ZWEITEN HÄLFTE DES 19. JAHRHUNDERTS. \\ Zu Theodor Storms Novelle „Hans und Heinz Kirch”
}

In der Zeit zwischen 1881 und 1888 schrieb Theodor Storm eine Reihe von späten Novellen, die bestimmt sind von einer scharfen Kritik an den historischen und sozialen Entwicklungen, die Storm aufmerksam beobachtet hat. Dabei rührt der Impuls des Schreibens aus der konkreten Lebenssphäre, nicht von einer Position theoretisierender und abstrakter Kritik her:

er entnahm seine Stoffe dem vertrauten Kreis von Haus, Heimat, Familie, was ihm den freundschaftlichen Vorwurf Theodor Fontanes einbrachte, er betriebe ,Husumerei'. ${ }^{1}$

Storms Intention folgt den Vorgaben der novellistischen Struktur. Die Texte sind jeweils konzentriert auf ein Problem, das in der vielberufenen 'unerhörten Begebenheit' prononciert vor Augen gestellt wird. Die Novelle soll sich in einem klar überblickbaren, gut konturierten Handlungsablauf mit einem zentralen Ereignis, der Problemstellung befassen. Die meisten Novellen sind in eine Rahmenerzählung eingebettet und stellen eine „unerhörte Begebenheit” dar.

Der Rahmen dient bei Storm zur Funktionsbestimmung des Erzählten; er stellt insbesondere eine Beziehung zwischen der erzählten Geschichte und ihrem nachzeitlichen Leser - Schreiber her. Die Art, wie dieser Leser dem Text aus der Vergangenheit begegnet, löst das Zukunftsdenken des Schreibers der alten Geschichte ein und zeigt, wie , entlegen' oder ,gegenwärtig' dessen Schicksal ist. $^{2}$

1 Van Rinsum, Annemarie und Wolfgang: Realismus und Naturalismus. In: Deutsche

Literaturgeschichte Bd. 7, Deutscher Taschenbuch Verlag, München, 1994. S. 159.

Husum ist die Stadt, in der er damals gelebt hat.

2 Aust, Hugo: Novelle. J. B. Metzlersche Verlagsbuchhandlung, Stuttgart, 1990.

S. 116.

Kairoer Germanistische Studien 11, 1998/99. 
Das Erzählen einer Geschichte aus der Vergangenheit ermöglicht so eine Perspektivierung nach vorn auf eine zweite Zeitstufe hin, vom Geschehen im Präteritum aus auf die Gegenwart oder einer näher oder entfernter liegende Zukunft. Der Rahmen dient aber nicht dazu, eine objektive Distanz zu gewinnen, sondern bewirkt gerade umgekehrt eine Intensivierung des subjektiven Blicks. Hier liegt trotz des engen Erzählhorizonts der ,Husumerei' und trotz des traditionell novellistischen Verfahrens das Moderne von Storm im Realismus. Er entobjektiviert die Darstellung.

"Das psychologische Interesse an vagen und unbestimmten Zuständen der Seele, an diffizilen Gebrochenheiten und an der schleichenden Tragik, die über große Zeiträume hinwegreichen kann, [.....], nimmt bereits manche Stilzüge moderner Prosa vorweg.“3 ${ }^{\text {“3 }}$ Das schließt die Vernachlässigung äußerer Kriterien für modernes Erzählen im späten Realismus ein.

Die ursprüngliche Lust am Fabulieren, und zwar in einer subjektiven Erzählweise, der es um die rätselvolle, vom Gefühl getragenen Beziehung zwischen Einst und Jetzt zu tun ist, läßt Komposition, Bewertung und Ironie zurücktreten. Die souveräne Verschleierungstechnik Kellers, die kluge Absichtlichkeit Meyers oder die Gelassenheit Stifters wird man hier vergeblich suchen. ${ }^{4}$

Das Verfahren Storms ist eben dieses: einen geographisch fixierten Ort zu fiktionalisieren und damit bedeutend zu machen. Dies ist bemerkenswert im Vergleich zu Keller, der seinen fiktionalen Ort Seldwyla so ausgestaltete, daß er für real gehalten wurde. Seit die erste Hälfte dieser Erzählungen erschienen, streiten sich etwa sieben Städte im Schweizerlande darum, welche unter ihnen mit Seldwyla gemeint sei; und da nach alter Erfahrung der eitle Mensch lieber für schlimm, glücklich und kurzweilig als für brav, aber unbeholfen und einfältig gelten will, so hat jede dieser Städte

3 Wiese, Benno von: Die deutsche Novelle. Von Goethe bis Kafka. Interpretationen II, Bagel, Düsseldorf. 20. Aufl., 1986. S. 216 f.

4 ebda. S. 218. 
dem Verfasser ihr Ehrenbürgerrecht angeboten für den Fall, daß er sich für sie erkläre. ${ }^{5}$

Keller sieht sich in der Lage Homers, der Troja beschrieben hat ${ }^{6}$. Also ist das Verfahren Storms geradezu gegenläufig zu dem, das Keller anwendet. Der eine geht vom realen Husum aus und entwirft eine fiktionale Stadt, und der andere setzt ein Wort als fiktionalen Ortnamen, und die Rezipienten suchen den realen Ort dazu. Das eine Mißverständnis läßt sich in dem Vorwurf „Husumerei” erkennen, während Keller das Mißverständnis ironisiert:

"Während aber einige der Städte hartnäckig fortfahren, sich ihres Homers schon bei dessen Lebzeiten versichern zu wollen, hat sich mit dem wirklichen Seldwyla eine solche Veränderung zugetragen, daß sich sein sonst durch Jahrhunderte gleich gebliebener Charakter in weniger als zehn Jahren geändert hat und sich ganz in sein Gegenteil zu verwandeln droht. “7

In Storms Prosa verringert sich die erzählerisčhe Distanz, die zum Gestus des Epischen, etwa bei Stifter oder Fontane, gehört. Es intensiviert sich die Lektüre so, daß der Leser an dem Geschehen teilzunehmen meint und an den dargestellten Erfahrungen teilhaben kann. Es gehören also das Subjektive und das Stimmungshafte des persönlichen Erzähltons zu Storms Erzählperspektive. Dies bestätigt Benno von Wiese:

"Der Erzähler selbst läßt sich in das Vorgangshafte stärker hineinziehen und verführt auch seinen Leser dazu. ${ }^{\text {"8 }}$

Es bedeutet dies eine intensivere Konfrontation mit der erzählerisch vermittelten Realität. In Storms Novelle "Hans und Heinz Kirch” (1881/82) erscheint das deutlich. Sie spielt gegenwartsnah in einer kleinen Stadt an der Ostsee, mit starker Prägung durch die kulturelle und geographische Situation

5 Keller, Gottfried: Die Leute von Seldwyla. Einleitung. Zweiter Teil. Wilhelm Goldmann Verlag, 4 Aufl. 1983. S. 269.

6 Vgl. Czucka, Eckehard: Emphatische Prosa. Franz Steiner Verlag, Stuttgart 1992. S. $108 f$.

7 Keller, Gottfried: Die Leute von Seldwyla. a.a. O. S. 269.

8 Wiese, Benno von: Die deutsche Novelle. a.a.O. S. $220 \mathrm{f}$. 
des norddeutschen Raumes. Der fiktionale Ort dieser Erzählung stimmt also in vielen Einzelheiten mit Storms realem Husum überein.

In den frühen Erzählungen Storms wie "Auf dem Staatshof" (1856/58), „Im Schloß” (1861), „Drüben am Markt” (1860), „Abseits” (1863) und "St. Jürgen” (1869) erhält sich auf solchem Wege noch ein biedermeierliches Bild von einer Welt der Behaglichkeit im Kleinen. In den späten Erzählungen ist die familiär und auch kleinbürgerlich enge Lebenssphäre der einzige schützende und orientierende Raum, von dem sich der einzelne niemals ohne gefährliche seelische oder sittliche Erschütterungen lösen kann. Aber gerade in diesen privaten Raum sind dann auch zentrale Konflikte eingelagert, zwischen Vater und Sohn, Bruder und Bruder, Vater und Tochter oder überhaupt im Verhältnis der nächsten Generation zur früheren und zu den alten Traditionen. Das bedeutet für Storms Erzählungen aber nicht, daß die Normen nach außen zu verlagern sind, in weiter zu ziehende Kreise, sondern daß es gar keine Normen mehr gibt, wenn der innere Zusammenhalt verloren ist. Storm hat deswegen nicht nur den Untergang junger Menschen am Starrsinn und tyrannischen Willen der Väter ("Der Herr Etatsrat”, "Hans und Heinz Kirch”) geschildert, sondern auch die Zerstörung einer ganzen Familie durch den Leichtsinn oder den Zorn eines einzelnen ("Carsten Curator”, "Zur Chronik von Grieshuus”).

Diese Tendenz hat Storm beibehalten, obwohl er die ganze Reife seiner Kunst eigentlich erst in seiner Spätzeit zur Geltung bringt. Er erlaubt sich den Griff nach den verschiedenen Stoffen, die meist tragisch und rührend sind. In seiner Untersuchung zur Literatur des bürgerlichen Realismus beschreibt Andreas Huysseen Storms Entwicklung in der Novelle:

"Storm kam von den lyrisch stimmunghaften Situationsnovellen der Frühzeit (um 1850) über psychologisch-erotische Problemnovellen (1857-67), deren künstlerischer Wert oft durch Anpassung an Heyses Novellenschema beeinträchtigt ist, zu den tragischen Schicksalsnovellen der Spätzeit (nach 1871), bei denen zwischen historischen Novellen wie "Aquis submersus" und

9 Vgl. Stuckert, Franz: Theodor Storm. Der Dichter in seinem Werk. Max Niemeyer

Verlag, Tübingen, 3. Aufl., 1966 S. 127 f. 
einer mehr zeitbezogenen Novellistik wie in "Hans und Heinz Kirch” zu unterscheiden ist. ${ }^{\text {“10 }}$

Diese Perspektive schließt ein, daß durchaus die großen Konflikte der Zeit ihren Ausgang in scheinbar privaten Konstellationen finden. Der gesellschaftlich vermittelte Konflikt erscheint als individueller, z.B. eben als Generationskonflikt in der Novelle "Hans und Heinz Kirch”. Der Titel, der ein unspezifiziertes Verwandtschaftsverhältnis anzeigt, bis zum gleichen Anlaut der Vornamen, enthält so schon das Programm. Damit kann sich Storm zugleich aber "dem Zwang zur Psychologisierung, gegen den er sich selbst wendet, $[\ldots . .$.$] nicht entziehen." 11$

Allerdings versucht Storm, diesem Psychologischen dadurch Unbedingtheit $\mathrm{zu}$ geben, daß er die individuelle Disposition zu einer schicksalhaften Konstellation überhöht. Er entwickelt ein Konzept von Novelle, mit dem die alte Notwendigkeit des Tragischen nun in der Novelle wirksam werden soll. Storm schreibt:

"die heutige Novelle ist die Schwester des Dramas und die strengste Form der Prosadichtung. Gleich dem Drama behandelt sie die tiefsten Probleme des Menschenlebens. ${ }^{\text {“12 }}$

So werden die Probleme des einzelnen und zwischen einzelnen, zwischen Hans und Heinz Kirch, gesteigert zum realistischen Allgemeinen.

10 Huyssen, Andreas (Hrsg.): Die deutsche Literatur. Ein Abriß im Text und Darstellung, Bd. 11 Bürgerlicher Realismus. Philipp Reclam jun, Stuttgart, durchgesehene und bibliographisch ergänzte Ausgabe 1978. S. 156.

11 Vincon, Hartmut: Theodor Storm. J.B. Metzlersche Verlagsbuchhandlung, Stuttgart. 1973. S. 64.

12 Storm, Theodor: Eine zurückgezogene Vorrede aus dem Jahre 1881. In: Theodor Storm. Sämtliche Werke. Bd. 4. Hrsg. v. Dieter Lohmeier. Deutscher Klassiker Verlag, Frankfurt/ Main. 1988. S. 409. 
Diese Probleme werden von Storm in seinen Spätwerken nicht mehr in der traditionellen Form der Novelle, mit Rahmen, Spukgeschichten und 'Falken' dargestellt, sondern es handelt sich um Symbole, die im Laufe der Novelle auftauchen.

Immer wieder wurde in den Novellentheorien von Goethe bis zu den Theoretikern des 20. Jahrhunderts auf den "Konflikt des Gesetzlichen und des Ungebändigten” als Mittelpunkt der novellistischen Gestaltung verwiesen. ${ }^{13}$ In der unterdrückten Vorrede zur Novelle aus dem Jahre 1881, aus der schon zitiert wurde, heißt es weiter, es verlange diese

"zu ihrer Vollendung einen im Mittelpunkt stehenden Konflikt, von welchem aus sich das Ganze organisiert." ${ }^{, 14}$

Diese Zentralisierung und Generalisierung des Konflikts ist in der Stormschen Perspektive als ein Aspekt des Realismus zu begreifen. Das hebt sein Werk hinaus über die formal verfahrende Novellistik von Zeitgenossen wie Paul Heyse und Otto Ludwig.

$\mathrm{Da}$ der Beitrag sich mit einem gesellschaftlichen, realistischen Problem befaßt, wurde eine Methode gewählt, die sich dazu eignet. Um dem kritischen Potential der Texte Storms gerecht werden zu können, müssen sie in einer hermeneutischen Analyse interpretiert werden. Erst eine solche hermeneutische Bemühung läßt sichtbar werden, welche Elemente der Verhältnisse in der Darstellung von Bedeutung sind, welche aber als minder wichtig gehalten werden. Auf der Basis einer solchen Interpretation kann dann das kritische Potential der Novelle gewürdigt und angemessen beurteilt werden. Diesen Versuch unternimmt dieser Beitrag am Beispiel der Darstellung des Generationskonflikts in der Novelle „Hans und Heinz Kirch”.

13 Vgl. Kunz, Josef: Die deutsche Novelle im 19. Jahrhundert. Erich Schmidt Verlag, Berlin, 1970 S. 13.

14 Storm, Theodor: Eine zurückgezogene Vorrede aus dem Jahre 1881. a.a. O.

S. 119. 
Storm hat die ersten Entwürfe zu seiner Novelle "Hans und Heinz Kirch" schon während seines Aufenthaltes bei seiner Tochter Lisbeth in Heiligenhafen im Oktober 1881 fertiggestellt. Die Quelle der Novelle stammt aus einer Chronik, die der Hauptpastor von Heiligenhafen, Heinrich Scholtz, verfaßt hatte und die dem Dichter offenbar im Pastorat, im Hause seines Schwiegersohnes, zugänglich war. Die erzählte Geschichte ist also auch historisch bezeugt. Daneben hat Storm auch die alte, aus dem Jahre 1743 stammende sogenannte "Nachricht von der Stadt Heiligenhafen”, eine Stadtchronik, als Vorlage benutzt. ${ }^{15}$ Karl Ernst Laage weist darauf hin, daß Storm in seinem Tagebuch "Was der Tag giebt" an verschiedenen Stellen auf die Chronik verwiesen und manchmal sogar aus ihr zitiert hat. Storm ist demnach sehr genau dem Material gefolgt und hat dem Erzähler einen streng mimetischen Charakter geben wollen, darin streng das Realismuspostulat beachtend. Noch die Namen der beiden Hauptgestalten sind hierdurch angeregt. Denn in der Chronik von Scholtz werden als "Raths-Verwandte [Senatoren] der Stadt" ein Hans Karck und ein Hinrich Karcke genannt. "Karck” ist - nach Laage - die plattdeutsche Form von Kirche. ${ }^{16}$ Laage weist auf mehrere Stellen der Chronik hin, die Storm dann fast zitierend übernommen hat. Der Schauplatz der Geschichte von dem Schiffer Brandt und seinem Sohn Christian - dies sind die Namen der historischen Vorbilder von Hans und seinem Sohn Heinz - ist die Stadt Heiligenhafen. Storm hat eine ähnliche Kleinstadt zum Schauplatz gemacht, ohne sie mit einem Namen zu nennen. ${ }^{17}$

15 Vgl. Laage, Karl Ernst: Nachricht von der Stadt Heiligenhafen (1743). Eine bisher unbekannte Quelle zu Storms „Hans und Heinz Kirch”. In: Schriften der Theodor-Storm-Gesellschaft. Hrsg. v. K.E. Laage und Volkmar Hand. Westholsteinische Verlagsanstalt Boyens \& Co., Heide in Holstein, Schrift 26, 1977 S. 72. 
In der Novelle "Hans und Heinz Kirch” sind die Positionen von Vater und Sohn sehr unterschiedlich. Dem einen ist es nur um die äußere Lebenssicherung, den Stand und das Ansehen von außen her zu tun, der andere verlangt nach eigener, innerer Bestimmung. In der Art, wie beide miteinander den Konflikt austragen oder auch mit Schweigen übergehen, sind sie sich so ähnlich wie eben Vater und Sohn, engstirnig bis zur Hartherzigkeit, kompromißlos.

Storm beginnt seine Darstellung des Konflikts schon mit der Beschreibung des Ortes, an dem die als Novelle dargestellten Geschehnisse sich abspielten. Die kleine Stadt an der Ostsee wird zur Metapher des harten, verschlossenen Lebens.

"Gleichwohl, wer als Fremder durch die auf- und absteigenden Straßen der Stadt wandert, wo hie und da roh gepflasterte Stufen über die Vorstraße zu den kleinen Häusern führen, wird sich des Eindrucks abgeschlossener Einsamkeit wohl kaum erwehren können, zumal wenn er von der Landseite über die langgestreckte Hügelkette hier herabgekommen ist. ${ }^{\text {"18 }}$ (HHK 58)

Weiter wird angegeben, da $\beta$ die Bürgerglocke gleichzeitig mit der Glocke des Kirchturms abends um zehn schlägt und daß nach dieser Zeit kein Knabe oder Sohn der Stadt mehr auf den Straßen gesehen werden darf. Die Menschen schließen sich also ein. Im Fortgang der Erzählung erscheint dieses Verhalten als Bedingung dafür, daß sie dem schweren Leben überhaupt standhalten können.

"Aber in der kleinen Stadt leben tüchtige Menschen, alte Bürgergeschlechter [...]“" (HHK 58)

Storm will hier mit den Mitteln novellistischen Erzählens andeuten, daß diese Strenge durchaus nötig ist, um tüchtige und strebsame Bürger zu

18 Storm, Theodor: Hans und Heinz Kirch. In: Sämtliche Werke. Novellen 1881 -

1888. Hrsg. v. K.E. Laage und Dieter Lohmeier. Bd.3. Deutscher Klassiker Verlag Frankfurt/M, 1988. S.58.

Im folgenden Nachweise dieser Ausgabe im fortlaufenden Text mit der Sigle HHK Seitenzahl. 
erziehen. Der Vater Hans gehört zu dieser kleinen strengen Gesellschaft, behandelt seinen Sohn jedoch noch extremer, als es in der Stadt ansonsten üblich ist. Der Vater sticht also durch besondere Härte ab. Er erfüllt das Gesetz des Lebens in solcher Konsequenz, mit starken Zügen innerer Verhärtung, daß er sich dann doch wieder von den anderen in der Stadt unterscheidet. Er hat durch rechtschaffene Arbeit eine angesehene Stellung unter seinen Mitbürgern errungen, aber in seiner Kälte hat er sich allem Familiären zugleich entfremdet.

Schon am Anfang der Novelle erfährt der Leser vom Geiz des Vaters, der mit seiner Frau schimpft, wenn sie dem Jungen am Werktag eine Süßigkeit kauft:

\section{"All wedder'n Dreling umsunst utgeb'n!"” (S.61)}

Diese extreme Strenge und dieser Geiz werden selbst von der kleinbürgerlichen, zum Teil auch konservativen Gesellschaft des Ortes ironisch aufgenommen. Sie macht diese Schimpfrede des Vaters vom verschwendeten Dreier bei jener Gelegenheit zum Sprichwort für die Strenge des Hans Adam Kirch:

“ [...] Dies geflügelte Wort lief einmal durch die Stadt; Hans Adam hatte es seiner Frau zugeworfen, als sie ihrem Jungen am Werktag einen Sirupuskuchen gekauft hatte." (HHK 61)

Hans wird weiter von Storm als ein genauer, zuverlässiger Kapitän und gewissenhafter Geschäftsmann beschrieben. Der Sohn Heinz ist im Aussehen ein Ebenbild des Vaters, sagen die Leute, und so will ihn Vater Hans auch innerlich als sein zweites Ich haben. Seine eigene, gradlinig in der vorbestimmten Bahn geführte Existenz will er im Sohn verlängern. Hier entsteht ursächlich nämlich die Auseinandersetzung zwischen dem Vater und dem Sohn. Der Sohn ist eben nur äußerlich dem Vater ähnlich. Er liebt das

19 Ubersetzung vom Plattdeutschen: „Schon wieder einen Dreiling umsonst ausgegeben!" 
sympathische Mädchen Wieb, das von den anderen Mädchen abgelehnt wird, da seine Mutter einen schlechten Ruf hat. In der Novelle wird kein Grund für den schlechten Ruf gegeben.

"Thre Mutter war die Frau eines Matrosen, eine Wäscherin, die ihr Kind sauberer hielt als, leider ihren Ruf." (HHK 65)

Damit wird erkennbar, daß der Sohn sich über Konvention und Vorurteile hinwegsetzt. Diese Strenge der Gesellschaft, die Strenge des Vaters kann Heinz, als er zu einem jungen Mann herangewachsen ist, nicht mehr akzeptieren. Die Liebe zu jenem Mädchen ist im Grunde schon die Entscheidung für eine Gegenexistenz zum väterlichen Leben.

Heinz soll als Siebzehnjähriger auf eine einjährige Reise mit dem Hamburger Schiff "Hammonia" gehen. Er folgt dem väterlichen Befehl. Aber dann kommt der Junge nicht zurück, und man erhält keine Briefe von ihm. Am Abschiedsabend hatte Hans furchtbar mit Heinz geschimpft, nur weil der Sohn zu spät nach Hause gekommen war. Hans meint aber später:

"Er wird schon kommen,..., er weiß gar wohl, was hier zu Haus für ihn zu holen ist.“(HHK 76)

Hans war zu stolz, dem Sohn vor seiner Fahrt noch anders zu begegnen, und Heinz ist zu stolz, zu diesem Vater zurückzukehren.

Das materielle Leben ist denn auch für Hans der einzig gültige Maßstab, und er glaubt, daß sein Sohn ebenfalls dieser Norm folgen werde. Er denkt sich zu dem aus, daß Heinz in der Ferne ein in seinem Sinn erfolgreiches Leben führe und ihn schließlich überraschen werde, indem er als ein wohlhabender, erfolgreicher Seemann zurückkehren werde. Hans wartet auf diesen Moment und ist sehr enttäuscht, als nach zwei Jahren endlich ein unfrankierter Brief eintrifft; die fehlende Briefmarke versteht der Vater als Zeichen, daß der Sohn also nicht einmal mehr das Porto bezahlen kann und nimmt den Brief nicht an. Die Novelle lenkt unsere Gedanken auf bestimmte Perspektiven. In diesem Zutrauen auf die Rückkehr des Sohnes liegt die ganze Trostlosigkeit des Verhältnisses beider beschlossen. Der Vater findet vor sich 
selbst keine anderen Gründe dafür, daß der verlorene Sohn zurückkommen werde, als eben materielle.

Wenn Heinz nach diesen Jahren ohne Erfolg zurückkommen will, dann soll ein solcher Sohn lieber fortbleiben. - So denkt jedenfalls der Vater:

\section{"Lump! [...] so kommst du nicht in deines Vaters Haus!"(HHK 80)}

In dieser sich nur in den Gedanken des Vaters vollziehenen Abweisung durch den Vater erreicht die Konfliktdarstellung ihren Höhepunkt. Mit dieser Zurückweisung bestraft Hans nicht nur Heinz, sondern auch sich selbst, seine Frau, seine Tochter und Wieb. Weder die Sorgen der Mutter um den Sohn, der Schwester um den Bruder, der Freundin um den Geliebten noch die begütigenden Worte eines gut zuredenden Pastors lassen Hans von seiner Verstoßung abstehen. Wie niemals im Leben, so kennt er auch hier kein Zurück. Die Mutter stirbt später, ohne ihren Sohn gesehen zu haben.

Als man nach 17 Jahren wieder aufeinander trifft, zeigt sich, daß Hans bereit ist, den Sohn wieder anzunehmen, wenn er sich in die ,Ordnung' schickt, also der Sohn wird, als den sich Hans seinen Heinz immer denken wollte. Aber der Sohn zeigt nicht die Bereitschaft zu solcher Anpassung, und das macht die Trennung endgültig. Die Fremdheit ist überdies schon so groß, daß der Vater auf Gerüchte zu hören beginnt, daß der Heimgekehrte gar nicht sein Sohn, sondern vielmehr ein anderer sei, der sich für seinen Sohn bloß ausgebe. Und tatsächlich ist der Sohn ein anderer, nur äußerlich sich selbst und damit dem Vater gleich. Allein Wieb kann Heinz wirklich wiederkennen, weil sie ihn immer schon als den liebte, der er selber ist. Als sie davon mit dem Vater spricht, entgegnet dieser nur:

"Nun, Wieb, wenn's denn dein Heinz gewesen ist, es ist nicht viel geworden aus euch beiden (S.121)“

Daß Hans nur "dein Heinz" und nicht "mein Heinz” oder überhaupt nur den bloßen Namen sagt, zeigt seine Ablehnung dieses Sohnes.

Auf die Bitten der Tochter und Schwester, Heinz zurückzuholen, ist die so einfache wie grundsätzliche Antwort: 
"mein Heinz hat schon vor siebzehn Jahren mich verlassen"(HHK 122)

Hans Kirch wird da gar nicht mehr als Vater beschrieben, sondern nur noch als das "tote Bild" eines Menschen. ${ }^{20}$

So schildert uns Storm die Figur eines herzlosen Vaters, der kein Verständnis, keine Gefühle und auch keine Hoffnungen für sein eigenes Blut hat. Hans will keinen Zwischenweg finden, entweder macht der Sohn, was der Vater für ihn geplant hat, oder er wird in die Verbannung geschickt, indem er aus demVaterhaus gewiesen wird.

Storm zeigt den Vater als einen Menschen, dem die menschlichen Beziehungen und Gefühle vollkommen fehlen. Der Sohn hatte das schon früh geahnt, und deshalb ist er ohne Hoffnung auf eine Versöhnung einfach fortgegangen. Die Selbsttäuschung des Vaters reicht bis zu bewußter Wahrnehmungsverweigerung,

"glaubte er doch selber nur den Erben seiner aufstrebenden Pläne in dem Sohn zu lieben" (HHK 64)

Storm zeigt in dieser Novelle, daß der Vater eigentlich am Scheitern des Sohnes schuldig ist. Hans will das nicht wahrnehmen, denn das Scheitern des Sohnes würde auch das Scheitern seines eigenen Lebens bedeuten, das letztlich auf das Fortleben in dem anderen gerichtet bleibt. In dem Augenblick, in dem der Vater endgültig spürt, daß der Sohn einfach nicht das vorgestellte Ideal erfüllen kann, fallen sein ehrgeiziges Planen und Handeln in sich zusammen. In seinen letzten Lebensjahren arbeitet Hans nicht mehr, er gibt sich und alles auf. Das ganze Vermögen, das Ergebnis langer Jahre und harter Arbeit, wird jetzt dem Schwiegersohn überlassen.

Die Frage, ob es überhaupt noch der richtige Sohn Heinz Kirch ist, den Hans nach 17 Jahren vor sich sieht, kennzeichnet schon das grundsätzliche Mißtrauen und markiert eine letztlich unüberwindbare Kluft. Heinz wird denn

Vgl. Storm, Theodor: Hans und Heinz Kirch a.a.O. S.121. 
auch als eine fremde Erscheinung dargestellt, kritisch von allen beäugt, eben selbst vom eigenen Vater. Im äußeren Fremdsein erscheint das innere. Das nimmt der gefühlvolle Sohn sehr wohl wahr, und deshalb weiß er sich auch als der wirkliche Sohn trotzdem als der falsche. Er geht erneut fort.

Der Sohn hat aber nicht alles Recht auf seiner Seite. Er ist (sein eigenes Scheitern, seine erfolglose, im Grunde elende Existenz 'außerhalb' zeigt dies) an die Grundwerte gebunden, die das Leben der Gemeinschaft, aus der er kommt, tragen. Storm hat in seinen Werken immer wieder versucht, die Lebenswerte der deutschen bürgerlichen Gesellschaft darzustellen. Franz Stuckert schreibt dazu:

"Storm lebt so tief im Ethos seines Volkes, daß sein ganzes Werk eine im Vergleich mit Raabe muß man sagen - völlig problemlose Darstellung der deutschen Charakterwerte enthält. “21

Die Erzählung ist darin allerdings weiter als der Autor in seiner generellen Auffassung selbst. Denn sie zeigt ja, daß jene Grundwerte kein ewig verbürgtes An-sich haben. Der Sohn kann deshalb nicht zurückkehren in den von überlieferten Werten bestimmten Lebensbereich, weil der Vater den Lebensbereich zerstört hat, weil also die Tradition im Grunde sich selbst zerstört hat. Dies wird sehr deutlich schon darin gezeigt, wie der Vater seine Existenz zu begründen suchte. Es ist geradezu die Existenzzerstörung durch die Übersteigerung des Prinzips der Existenzbegründung.

Der Vater Hans ist ein tüchtiger, strebsamer Kleinbürger. Durch seinen Fleiß und seine Sparsamkeit ist er zum Schiffsbesitzer geworden:

"Mit unermüdlichem Tun und Sparen hatte er sich vom Setzschiffer zum Schiffseigentümer hinaufgearbeitet; freilich war es nur eine kleine Jacht, zu der seine Mittel gereicht hatten, aber rastlos und in den Winter hinein, wenn schon alle anderen Schiffer daheim hinter ihrem Ofen saßen, befuhr er mit seiner Jacht die Ostsee." (HHK 60)

21 Stuckert, Franz: Theodor Storm. Der Dichter in seinem Werk. Max Niemeyer

Verlag, Tübingen. 1966. 3. Aufl., S.137. 
"Weiter wird von Storm die Rastlosigkeit des Bürgers Hans beschrieben. Seine Sparsamkeit betrifft auch seine ganze Familie, besonders seine Frau, die

nur seinen Willen kannte und überdies aus Furcht vor dem bekannten Jähzorn ihres Mannes sich das Brot am Munde sparte, so pflegte dieser bei jeder Heimkehr auch zu Hause einen hübschen Haufen Kleingeld vorzufinden." (HHK60)

In so einer Familie, in der keine normale Ehe geführt wird, also in einer personalen Beziehung, in der Liebe und Gefühle keine Rolle spielen, sondern in der Pfennigfuchserei und Jähzorn des Mannes bestimmend sind, ist der junge Heinz aufgewachsen. Die Beziehung zwischen dem Vater und der Mutter war wie die Beziehung zwischen einem absoluten Herrscher und seinen unterdrückten, hilflosen Untertanen: ein strenger, sparsamer, geiziger, dazu auch zorniger Vater und eine liebe, ängstliche und hilflose Mutter. So sind die Verhältnisse lange schon deformiert, bevor Heinz es in ihnen nicht mehr aushalten kann. Durchaus ist es eine Form von Liebe, von väterlicher Liebe, in der sich das Tragische entfaltet. Hans lebt im Anfang der Novelle ganz für den Sohn Heinz. Storm erwähnt die Zärtlichkeit des Vaters und verfolgt ihre Entwicklung bis dorthin, wo die Sorge für den Sohn aufgesaugt wird von den Sorgen, die sich der Vater, eben für den Sohn und um dessen Zukunft sich sorgend, um die Existenzsicherung macht.

"Was Hans Kirch an Zärtlichkeit besaß, das gab er seinem Jungen; bei jeder Heimkehr lugte er schon vor dem Warder ${ }^{22}$ durch sein Glas, ob er am Hafenplatz ihn nicht gewahren könne; kamen dann nach der Landung Mutter und Kind auf Deck, so hob er zuerst den kleinen Heinz auf seinen Arm, bevor er seiner Frau die Hand zum Willkommen gab.“ (HHK 61)

An einem kleinen, aber markanten Ereignis wird deutlich, wie die väterliche Sorge ins Qualitätslose umschlägt. Einmal hat der Vater den Sohn

22 Warder: Werder, der, selten das; -s, - (Flußinsel; Landstrich zwischen Fluß u. stehenden Gewässern). 
als Sechsjährigen mit auf eine Fahrt genommen. Der Junge ist auf den Bugspriet $^{23}$ gestiegen, als der Vater seinen Mittagsschlaf hält.

"Hans Kirch wurde blaß wie der Tod; denn dort, fast auf der äußeren Spitze, hatte er seinen Heinz erblickt" (HHK 62)

Als der Sohn wieder sicher aufs Verdeck zurückgesprungen ist, ruft der 'gefahrgewohnte[n] Mann' mit zitternden Zähnen:

"Heinz, Heinz, das tust du mir nicht wieder! Krampfhaft preßte er den Knaben an sich; aber schon begann die überstandene Angst dem Zorne gegen ihren Urheber Platz zu machen" (HHK 63)

Das ist ein gerechter Zorn, daß nichts mit dem früheren Jähzorn zu tun hat, der aber dem Sohn trotzdem viel Schrecken vor dem Vater einjagt. Im nächsten Frühjahr will Hans den Sohn wieder mit aufs Schiff nehmen, aber der versteckt sich nun und muß mit Gewalt an Bord gebracht werden. Dabei ist das kleine Abenteuer des Sohnes ein Ausdruck seines Freiheitswillens gewesen. Auf der anderen Seite verschwindet die Angst des Vaters um den Sohn, die sich im Verbot ausdrückt, schon hier und in der Zukunft immer mehr hinter der Vielzahl der Verbote und Gebote selbst, die das Verhalten des Vaters gegenüber dem Sohn bestimmen.

"Die Zärtlichkeit des letzteren [Hans] kam gleicherweise immer seltener zu Tage, je mehr der eigene Wille in dem Knaben wuchs" (HHK 63)

In dem Moment also, in dem der Sohn aus dem Willen des Vaters heraustritt und seinen Weg zu gehen sucht, verwandelt sich die väterliche Liebe in väterliche Strenge, die schließlich in ganz und gar unväterliche Härte umschlägt.

Das Arbeiten an dieser Novelle ist Storm sehr schwer gefallen. Es war, wie er Keller in einem Brief mitteilt, von "eine[r] kräftige[n] tragische[n] Erschütterung" ${ }^{24}$ bestimmt. An Heyse schreibt Storm über diese Novelle:

23 Bugspriet, das u. der, - [e]s, -e (Seemannsspr. über den Bug hinausragende Segelstange). 
"Wo zwei solche Naturen als Vater und Sohn sich gegenüber in die Welt gesetzt sind und der Schlag in Folge ihrer Eigenheit erfolgt, [haben wir] das große Schicksal. ${ }^{\text {‘2s }}$

Was eigentlich der Sohn Heinz von dem Vater geerbt hat, ist die Hartnäckigkeit, nicht die Lebenseinstellung. Der Sohn Heinz könnte nur mit völliger Selbstaufgabe den Ansprüchen des Vaters genügen. So bietet ihm die Welt, aus der er kommt, keine Überlebensmöglichkeit. Aber die Welt, in die er geht, bietet eine solche Möglichkeit auch nicht. Hier ist und bleibt er der Fremde auf unsicherem Boden, ein Mensch in fortdauerndem Exil. Mit der Freiheit vom väterlichem Zwang, im Leben 'draußen', drohen ihm auch Ortlosigkeit und Scheitern. Und es hängt mit seiner eigenen Hartnäckigkeit zusammen, daß er sich dies lange nicht eingestehen will.

Husum, Storms Heimatstadt, wird zum Modell und zur Metapher für eine kleine, geschlossene Stadt am Meer. Diese wird von der Erzählung zum Symbol für Heimat als geschützten Raum erhoben. Der Kampf der Stadt gegen das Meer als Naturelement vertritt symbolisch die Anstrengungen, mit denen menschliche Existenz sich ihre Ordnung und lebbaren Bedingungen schafft in einem im ganzen nur noch als chaotisch vorstellbaren Unraum.

"Auf einer Uferhöhe der Ostsee liegt hart am Wasser hingelagert eine kleine Stadt, deren stumpfer Turm schon über ein Halbjahrtausend auf das Meer hinausschaut" (HHK 52)

"Die Menschen, die in dieser Stadt wohnen, leben in "abgeschlossener Einsamkeit“" (HHK 58)

24 Storm, Theodor: Theodor Storm - Gottfried Keller. Briefwechsel. Hrsg.

K.E.Laage. Kritische Ausgabe. In Verbindung mit der Theodor-Storm-Gesellschaft.

Erich Schmidt Verlag, Berlin, 1992. Brief an Keller am 27.11.1882.

25 Storm, Theodor: Sämtliche Werke in vier Bände. Hrsg. v. Peter Goldammer. 4. Aufl., Bd. 3, Berlin, Weimar, 1978. S. 743. 
Obwohl aber Storm hier vieles beinahe dokumentarisch festhält und strikt realistischen Darstellungspostulaten folgt, bis in die genauen Einzelheiten der erzählten Geschichte, verwandelt sich in der Novelle doch gleichzeitig der Kontext 'Husum' 'unrealistisch', aber eminent literarisch, in einen Ausdruck von Lebenssphäre, Heimat überhaupt. Im Grunde haben alle Menschen so wie die in Husum zu leben, wenn sie denn nicht wie Heinz als Verirrte und sich Verirrende umhergetrieben werden. Sobald man von seiner Heimat weggeht, lebt man unsicher, wie schwer auch das Leben im Eigenen sein mag. Heinz lebt fern von Husum ohne Identität. Förmlich jeder einzelne seiner Schritte schon bringt die Gefahr mit, in völlige Orientierungslosigkeit zu fallen,

"ich kann noch nicht wie ihr; muß mich immer erst besinnen, wo ich hinzutreten habe; ihr kennt das nicht, ihr alle nicht, Schwester!"(HHK 98)

Diejenigen, die ihre Heimat nicht verlassen haben, kennen weder solche Unsicherheit noch solche Vorsicht. Aus der Perspektive von Heinz ist es da fast schon eine Lebensleistung, nicht völlig zugrunde gegangen zu sein. Das Scheitern, wo es ihn doch nicht ganz zerstört hat, ist fast schon ein Erfolg, 'draußen' nämlich überlebt zu haben.

Heinz - darauf hebt die Novelle $a b$ - ist genauso hartnäckig wie sein Vater. Er lehnt die strenge Traditionen der Heimatstadt ab, er lehnt die Lebenseinstellung und die Zukunftspläne des Vaters ab und ist zu stolz, mit dem Vater darüber in eine Auseinandersetzung einzutreten. Das bleibt auch bei der Rückkehr nach 17 Jahren noch so. Der Vater redet nicht wirklich mit dem Sohn und der Sohn nicht wirklich mit dem Vater:

Eine Unterredung zwischen Vater und Sohn fand weder nach dem Kirchgang noch am Nachmittage statt. Am Abend zog Frau Lina den Bruder in ihre Schlafkammer: "Nun, Heinz, hast du mit Vater schon gesprochen?” Er schüttelte den Kopf: „Was soll ich mit ihm sprechen, Schwester”.(HHK 97)

Als ihm der Vater die Geschäftsbücher zeigt, damit er von dem gegenwärtigen Stande des Hauses als eines Handelsunternehmens Kenntnis 
nimmt, legt Heinz sie nach kurzen Hin- und Herblättern wie etwas Fremdes beiseite. Da heißt es auch:

"an eine Ausnutzung seiner Sohnesrechte schien der Heimgekehrte nicht zu denken"(S.94)

Heinz nimmt am väterlichen Leben nicht teil und ist damit auch in der Heimat noch in der Fremde.

Der Vater trägt den zweiten Vornamen Adam.

“ $\mathrm{Zu}$ diesen strebsamen Leute gehörte Hans Adam Kirch”(S.60)

Das macht ihn zum ersten Vater, zum Vater überhaupt. So ist auch der Konflikt zwischen Hans und Heinz Kirch exemplarisch für den Streit zwischen den Generationen seit je, wie 'Husum' für Lebensort seit je steht. Allerdings bedeutet Adam auch, der erste zu sein, der aus dem Paradies vertrieben wurde. Er ist schon demnach auch der erste, der nach dem Gottesfluch das Brot im Schweiße seines Angesichts zu essen hat. Wie der Sohn den Vater deshalb verläßt, weil er an diesem nichts Heimatliches hat oder erkennt, das ihn halten könnte, so hat der Vater die Heimat eigentlich bereits in sich selbst verloren. Die idyllische Welt von Husum hat sich diesem Vater bereits zur Welt des harten und härtesten Lebens verfremdet.

Storm stellt den Vater Hans Adam als eine Person dar, die rücksichtslos gegen sich selbst und gegen andere den Prinzipien dieses harten Lebens folgt und damit selbst das zerstört, was er sich und seinem Sohn zu erhalten trachtet. Der Zweck seines Lebens ist allein schon die Sicherung der Existenz geworden. Weil er dafür alles andere zweitrangig werden läßt, verwandelt er sich von einem Vater zu einem Unvater. Nur äußerlich gelingt ihm noch sein Leben; 'innen' hat er alles verloren; eben auch den Sohn. Und die letzten Lebensjahre bringt er wie ein schon Toter dahin.

"Aber Hans Kirch saß wie ein totes Bild; sein jahrelang angesammelter Groll ließ ihn nicht los; denn erst jetzt, nach diesem Wiedersehen mit dem 
Heimgekehrten, war in der grauen Zukunft keine Hoffnung mehr für ih" ". (HHK 121)

Sohn und Vater sind beide beschädigte Figuren, gerade darum sind beide aufeinander verwiesen und angewiesen; in ihrer hartnäckigen Selbstverschränkung als letzter Form von Fremdheit, völliger Isolation des Subjekts bleiben sie aber auch unweigerlich gegeneinander gestellt. Nur anderen gegenüber kann Heinz bemerkbar machen, daß es ihn zum Vater zieht, der Schwester gegenüber oder vor einem Jungen, der wie in einem Märchen davon träumt, nach Amerika zu gehen, um eine Wasserfrau zu heiraten:

"Tu das nicht, Karl; die Wasserfrauen sind falsch; bleib lieber in deines Vaters $\mathrm{Stor}^{26}$ und spiel mit deines Nachbarn Katze“ (HHK 95)

Mehr ist da für ihn selbst schon nichts mehr zu ändern. So sehr ist er der Fremdgewordene schon, da $\beta$ er wie mit einem Versehen auch in eine fremde Sprache fällt, bevor er eben wieder geht.

"Thanks for the alms and farewell for ever. Er wußte selbst nicht, warum er das nicht auf Deutsch geschrieben hatte." (HHK 118)

Selbst die Verwendung des Wortes 'alms' (Almosen) für Geld, das ihm der Vater überlassen hat, zeigt, in welcher Rolle Heinz sich selbst sieht.

Die Novelle stellt in Heinz Kirch die ausweglose Situation des Menschen im Ausgang des 19. Jahrhunderts dar. Dieser Mensch kann weder mit den Traditionen noch ohne sie leben. Ohne Vergangenheit ist er auch ohne Zukunft. Seine Vergangenheit ist der selbst schon gescheiterte Vater, seine Gegenwart und seine Zukunft sind sein eigenes gewisses Scheitern. Dabei ist es zu einseitig, die Schuld allein beim Vater zu suchen. So reagierte Gottfried Keller unmittelbar, nach einer ersten Lektüre.

26 Stor: Vom Englischen store = Lagerhaus abgeleitet. 
"Ich habe das Ende der Novelle schnell angesehen und muß nun noch das Zwischenschicksal des Sohnes erfahren. Die Wendung mit dem unfrankierten Brief ist ebenso schaıerlich ais verhängnisvoll. Man fühlt mit, wie wenn der aite Geldtropf ein Schiff voll lebendiger Menschen in die brandende See zurückstieße. ${ }^{‘ 2}$ ?

Ebensowenig genügt es aber, in dem Geschehen eine besondere tragische Überhöhung zu sehen, erklärbar aus spezifischen Bedingungen und einer unglücklichen Konstellation, obwohl dies Storms eigene Auffassung von der Novelle als 'Schwester des Dramas' zu bestätigen scheint:

"gleich dem Drama behandeit diese Novelle die tiefsten Probleme des Menschenlebens; denn der Vater-Sohn-Konflikt ist hier zu einem Prinzipalkonflikt zugespitzt, der naturnotwendig - unter den beengten Gegebenheiten einer kleinen Stadt und weil Vater und Sohn charakterlich ähnlich angelegt sind - zum tragischen Untergang leider führen muß. ${ }^{* 28}$

Im Grunde greift diese Novelle über diesen "Prinzipalkonflikt" hinaus, und Storm schildert überhaupt, unter welchen schwierigen Bedingungen, jenseits irgendwelcher Lösungen, am Ende des 19. Jahrhunderts zu leben ist.

Storms schwerer, ausdrucksvoller Erzähistil, läßt keine Relativierung des Geschehens zu, auch nicht die einer Sonderung zum extremen, aber darum doch einzelnen Fall. Es ist dem Dichter gelungen, eine bürgerliche Tragödie, darin zugleich eine allgemeine, in Prosa darzustellen. Der Konflikt der Generationen zeichnet weniger den Umbruch zwischen zwei Epochen nach, vielmehr bedeutet er die Ausweglosigkeit der jetzigen, im Ende des 19. Jahrhunderts, bestimmt von einem Traditionsverlust, den kein Zukunftsgewinn aufwiegt.

27 Keller, Gottfried: Theodor Storm - Gottfried Keller. Briefwechsel a.a.O. S.96.

Deutscher Klassiker Verlag. Frankf/M. Bd 3, 1988. S. 814. 
"Mit dieser Kritik an der bürgerlichen Gesellschaft und an der modernen Welt (die mit "Telegraphendrähten”, „Reichspost” und „Reichsgulden” unaufhaltsam in die kleine Seestadt vordringt) gehört die Novelle "Hans und Heinz Kirch” zu den ausdrucksstärksten realistischen Novellen Theodor Storms. Sie kündigt - wie Benno von Wiese hervorgehoben hat - "bereits den Geist eines neuen Zeitalters an".

Dies sind die Worte, mit denen Karl Ernst Laage seinen Kommentar über diese Novelle beendet.

Der Realismus wendet sich den sozialen Verhältnissen und ihrer Problematik zu. In der Darstellung der Probleme eines bürgerlichen Alltags entdeckt er die Ursachen der gesellschaftlichen Spannungen und Konflikte. Er versucht die schwierigen Folgen für den Einzelnen wie für die Gemeinschaft zum Vorschein zu bringen. Die literarische Gestalung lenkt den Blick weg von jeder sozialtechnischen Bewältigung der Konflikte und rückt die gesellschaftlichen Fehlentwicklungen in den Mittelpunkt novellistischer Darstellung.

Ebda. S. 815. 


\section{LITERATURVERZEICHNIS}

\section{PRIMÄRLITERATUR:}

aSTORM, Theodor: Hans und Heinz Kirch. Theodor Storm Sämtliche Werke.

Novellen 1881-88. Hrsg. v. Karl Ernst Laage und Dieter Lohmeier, Bd. 3. Deutscher Klassiker Verlag. Frankf./M. 1988.

₫STORM, Theodor: Eine zurückgezogene Vorrede aus dem Jahre 1881. In: Theorie und Kritik der Novelle von Wieland bis Musil. Hrsg. v. Karl Konrad Polheim. Tübingen. 1970.

mSTORM, Theodor: Theodor Storm - Gottfried Keller. Briefwechsel. Hrsg. v. K.E. Laage. Erich Schmidt Verlag; Berlin, 1992.

aSTORM, Theodor: Theodor Storm - Paul Heyse Briefwechsel. 3 Bde. Hersg. v. Clifford Albrecht Bernd. Erich Schmidt Verlag, Berlin 1970.

\section{SEKUNDÄRLITERATUR:}

1.AUST, Hugo: Novelle. J.B. Metzlersche Verlagsbuchhandlung, Stuttgart. 1990.

2.ALAM ELDIN, Hazim A.M.: Die literarische Darstellung des Kleinbürgertums in der Novellendichtung des 19. Jahrhunderts am Beispiel von Theodor Storm und Gottfried Keller. Magisterarbeit Al-Azhar Univ. Kairo. 1990.

3.BAUMANN, Gerhard: Storm im Geschichtsbild des Historikers. In: Schriften der Theodor-Storm -Gesellschaft. Hrsg. v. K.E. Laage u. Gerd Eversberg. Bd. 43. Westholsteinische Verlagsanstalt Boyens \& Co., Heide in Holstein. 1994. 
4.BERND, Clifford A.: Storm und der europäische Realismus des 19. Jahrhunderts. In: Theodor Storm und das 19. Jahrhundert. Hrsg. v. Brian Coghlan und K.E. Laage. Erich Schmidt Verlag. Berlin 1989.

5.BERND, Clifford Albrecht: Storms Literaturkritik - ein Weg zum neuen Verständnis seiner Dichtung. In Schriften der Theodor-Storm-Gesellschaft. Hrsg. v. K.E. Laage. Schrift 17. Westholsteinische Verlagsanstalt Boyens \& Co., Heide in Holstein, 1968.

6.CHOWANIETZ, Siegfried: Jung und Alt im Konflikt. Generationsprobleme im Leben und in ausgewählten Novellen Theodor Storms. Peter Lang Verlag, Bern. 1990.

7.COGHLAN, Brian: Theodor Storm - gestern, heute und morgen. In: Schriften der Theodor-Storm-Gesellschaft. Hrsg. v. K.E. Laage und Gerd Eversberg. Bd. 42. Westholsteinische Verlagsanstalt Boyens \& Co., Heide in Holstein 1993.

8.COGHLAN, Brian: Theodor Storms Novelle: eine Schwester des Dramas? In: Schriften der Theodor-Storm-Gesellschaft . Hrsg. v. K.E. Laage und Friedrich Heitmann. Bd. 38. Westholsteinische Verlagsanstalt Boyens \& Co., Heide in Holstein. 1989.

9. COGHLAN, Brian: Storms Novelleneingänge. Ein Beitrag zur Darstellung seiner späten Erzählkunst. In: Schriften der Theodor-Storm-Gesellschaft. Hrsg. v. K.E. Laage. Schrift 17. Westholsteinische Verlagsanstalt Boyens \& Co., Heide in Holstein, 1968.

10. CZUCKA, Eckehard: Emphatische Prosa. Das Problem der Wirklichkeit der Ereignisse in der Literatur des 19. Jahrhunderts. Sprachkritische Interpretation zu Goethe, Alexander von Humbolt, Stifter und anderen. Franz Steiner Verlag, Stuttgart. 1992. 
11. DE CORT, Josef: Die Rolle der Ethik in Storms epischem Werk. In: Schriften der Theodor - Storm - Gesellschaft. Hrsg. v. K.E. Laage. Schrift 17. Westholsteinische Verlagsanstalt Boyens \& Co., Heide in Holstein, 1968.

12. DOANE, Heike A.: Probleme der Kommunikation in Theodor Storms „Hans und Heinz Kirch”. In: Schriften der Theodor-Storm-Gesellschaft. Hrsg. v. K.E. Laage und Friedrich Heitmann. Schrift 33. Westholsteinische Verlagsanstalt Boyens \& Co., Heide in Holstein. 1984.

13. FRÜHWALD, Wolfgang: Der Enthusiasmus des Lebens Individuation und Psychologisierung in Theodor Storms späten Erzählungen. In: Schriften der Theodor-Storm-Gesellschaft. Hrsg. v. K.E. Laage und Friedrich Heitmann. Schrift 33. Westholsteinische Verlagsanstalt Boyens \& Co., Heide in Holstein. 1984.

14.GRIMM, Günter: Theodor Storm: Ein Doppelgänger. Soziales Stigma als ımodernes Schicksalk. In: Romane und Erzählungen des Bürgerlichen Realismus. Hrsg. v. Horst Denkler. Philipp Reclam jun. Stuttgart, 1980.

15.HERRMANN, Walther: Nachwort zu Theodor Storm, Hans und Heinz Kirch. Reclam Verlag, Stuttgart, 1958.

16.HERMES, Eberhard: Johan Wolfgang von Goethe „Novelle”. Ernst Klett Verlag für Wissen und Bildung. Stuttgart, Dresden. 1995.

17.HuYSSEN, Andreas: (Hrsg.) Bürgerlicher Realismus. Bd. 11. Philipp Reclam jun. Stuttgart. 1974.

18. JENS, Walter: (Hrsg.) Kindlers neues Literatur Lexikon. Theodor Storm. Verlegt Bei Kindler, München 1991.

19.KNÜFERMANN, Volker: Untersuchung zur sprachlichen Wirklichkeit der Novellen. „Im Nachbarhause links”, „Hans und Heinz Kirch” und „Der Schimmelreiter" von Theodor Storm. Diss. Univ. Münster. 1967. 
20.KOHLSCHMIDT, Werner: Die Dichtung Theodor Storms und der Schweizer Realismus. In: Schriften der Theodor-Storm-Gesellschaft. Hrsg. v. K.E. Laage. Schrift 17. Westholsteinische Verlagsanstalt Boyens \& Co. Heide in Holstein. 1968.

21.KunZ, Josef: Die deutsche Novelle im 19. Jahrhundert. Erich Schmidt Verlag, Berlin. 1970.

22.LAAGE, Karl Ernst: Nachricht von der Stadt Heiligenhafen (1743). Eine bisher unbekannte Quelle zu Storms „Hans und Heinz Kirch”. In: Schriften der Theodor-Storm-Gesellschaft. Schrift 26. Westholsteinische Verlagsanstalt Boyens \& Co., Heide in Holstein. 1977.

23. LAAGE, Karl Ernst: (Hrsg.) Im Sonnenschein. Hans und Heinz Kirch. Text, Entstehungsgeschichte, Quellen, Schauplätze, Abbildungen. Westholsteinische Verlagsanstalt Boyens \& Co., Heide in Holstein. 1976.

24. LAAGE, Karl Ernst: (Hrsg.) Die Schuld des Vaters in Theodor Storms Novelle. „Carsten Curator”. In: Schriften der Theodor-Storm-Gesellschaft. Bd. 44. Westholsteinische Verlagsanstalt Boyens, Heide in Holstein. 1995.

25. LOHMEIER, Dieter: Plädoyer für sozialpsychologische Untersuchungen über Theodor Storm. In: Schriften der Theodor-Storm-Gesellschaft. Bd. 42. Westholsteinische Verlagsanstalt Boyens. Heide in Holstein. 1993.

26. MARTINI, Fritz: Deutsche Literatur im bürgerlichen Realismus. 1848 1898. J.B. Metzlersche Verlagsbuchhandlung. Stuttgart. 3. Aufl. 1974.

27. PASTOR, Eckart: Die Sprache der Erinnerung. $\mathrm{Zu}$ den Novellen von Theodor Storm. Athenäum Verlag. Frankf./M. 1988.

28. PREISENDANZ, Wolfgang: Gedichtete Perspektiven in Storms Erzählkunst. In Schriften der Theodor-Storm-Gesellschaft. Hrsg. v. K.E. Laage. Schrift 17. Westholsteinische Verlagsanstalt Boyens, Heide in Holstein. 1968. 
29.PREISENDANZ, Wolfgang: Theodor Storm: Novellistik im Zeitalter des Romans. In: Theodor Storm und das 19. Jahrhundert. Hrsg. v. B. Coghlan und K.E. Laage. Erich Schmidt Verlag, Berlin 1989.

30.RINSUM, Annemarie und Wolfgang van: Realismus und Naturalismus. Deutscher Taschenbuch Verlag. München. 1994.

31.RITCHIE, J. M. : Theodor Storm und der sogenannte Realismus. In: Schriften der Theodor - Storm-Gesellschaft. Hrsg. v. K.E. Laage und Friedrich Heitmann: Schrift 34 Westholsteinische Verlagsanstalt. Boyens \& Co., Heide in Holstein. 1985.

32. SCHILliNG, Michael: Erzählen als Arbeit am kollektiven Gedächtnis. $\mathrm{Zu}$ Theodor Storms Novellen nach 1865. In: Euphorion. Hrsg. v. Wolfgang Adam. Bd. 89., Universitätsverlag C. Winter Heidelberg. 1995.

33. SCHMIDT, Heiner: (Hrsg.) Quellenlexikon der Interpretationen und Textanalysen. Personal- und Einzelwerkbibliographie zur deutschen Literatur von ihren Anfängen bis zur Gegenwart. Bd. 12. Verlag für Pädagogische Dokumentation Duisburg. 1987.

34. SCHUSTER, Ingrid: Theodor Storm. Die zeitkritische Dimension seiner Novellen. Bouvier Verlag Herbert Grundmann. Bonn. 1971.

35.STUCKERT, Franz: Theodor Storm. Der Dichter in seinem Werk. 3. Aufl., Max Niemeyer Verlag Tübingen. 1966.

36. VINÇON, Hartmut: Theodor Storm. J.B. Metzlersche Verlagsbuchhandlung. Stuttgart. 1973.

37. WAGENKNECHT, Christian: Johann Wolfgang Goethe, Novelle.Erläuterungen und Dokumente, Reclam Verlag, Stuttgart. 1982. 
38. WIESE, Benno von: Die deutsche Novelle von Goethe bis Kafka. Interpretationen I. August Bagel Verlag. Düsseldorf, 1956.

39. WIESE, Benno von: Die deutsche Novelle von Goethe bis Kafka. Interpretationen II. Pädagogischer Verlag Schwann-Bagel. Düsseldorf. 1982.

40. WILPERT, Gero von: Sachwörterbuch der Literatur. Alfred Körner Verlag. Stuttgart. 7. verbesserte u. erweiterte Auflage. 1989. 